\title{
Effect of progressive absence epilepsy and its treatment on hemodynamics and autonomic regulation of the heart rhythm
}

\author{
M.L. Mamalyga', L.M. Mamalyga² \\ ${ }^{1}$ Bakulev Scientific Center of Cardiovascular Surgery, Moscow, Russian Federation \\ ${ }^{2}$ Department of Human and Animal Anatomy and Physiology of Moscow Pedagogical State University, \\ Moscow, Russian Federation
}

\begin{abstract}
Objective. To study hemodynamics and autonomic regulation of heart rhythm in patients with progressive non-convulsive convulsive activity of the brain and its treatment in WAG/Rij rats with genetically determined absence epilepsy.

Methods. Studies were conducted on rats of different age groups of the WAG/Rij and Wistar lines. The following methods were used for the study: telemetric monitoring of EEG, ECG, aortic blood pressure, echocardiography.

Results. Progressive convulsive activity (CA) in WAG/Rij rats with absence epilepsy (AE) is accompanied by a violation of the autonomic regulation of the heart and an increase in repolarization intervals of the left ventricle. In 6-and 9-month-old animals, anticonvulsant therapy with Convulex contributes to the improvement of the vegetative regulation of the heart. However, in 12-month-old animals, a decrease in peak-wave activity by the Convulex does not improve the impaired cardiac regulation and does not reduce the high risk of the occurrence of life-threatening arrhythmias.

Conclusions. The decrease in convulsive activity with Convulex makes it possible to improve the autonomic regulation of the heart. However, this possibility persists as long as progressive AE does not exceed a certain threshold. Thereafter, a decrease in brain SWD activity with Convulex does not improve impaired cardiac regulation.
\end{abstract}

Keywords: absence epilepsy, anticonvulsant therapy, hemodynamics, heart rate variability

\section{INTRODUCTION}

The main features characteristic of non-convulsive (absence) epilepsy were first recorded on an electroencephalogram and described by Gibbs, F.A. et al. back in 1935 (cited by 1). Since then, the study of the fundamental principles of this problem has not only not lost its relevance, but also significantly expanded the areas of research. A characteristic manifestation of absence epilepsy (AE) is spontaneous generalized spike-wave activity (spike-wave discharges - SWDs) of the brain, caused by pathological processes in the neuronal thalamocortical network $(2,3)$. Although SWDs are not so long, however, they can be repeated hundreds of times a day, which seriously disrupts the functional state of the central nervous system. The disorders caused by this are not limited to the brain. Frequently they cause autonomic dysfunctions, leading to imbalance of cardiovascular system regulation $(4,5,6)$, accompanied by life-threatening arrhythmias, which are one of the main causes of sudden death $(7,8)$. According data of different authors the risk of sudden cardiac death in people suffering from epilepsy is 2 to 3 times higher than that of the general population $(9,10)$.

Although the causal connection between the brain and the heart has long been an obvious fact, however, the interdependence of cerebral and cardiac disorders in the process of AE progression, like other types of epilepsy, remains insufficiently studied. Opinions of the authors about the dysfunctions of the heart arising on the background of non-convulsive epilepsy, and especially in the treatment of 
convulsive states, often diverge $(6,11,12,13,14)$. Most often, clinically severe dysfunction of the cardiovascular system occurs after progressive convulsive activity (CA) of the brain reaches a sufficiently high level. The underestimation of cardiac changes in the early stages of epileptogenesis adversely affects the prospects for recovery of patients with AE. The study of the fundamental mechanisms of interdependent disorders in the brain and heart in neurological diseases involves not only clinical, but also preclinical experimental studies on adequate models of laboratory animals.

\section{Study objective}

To study the functional features of hemodynamics and autonomic regulation of heart rhythm with the progression of non-convulsant convulsive activity of the brain and its treatment in WAG/Rij rats with genetically determined absence epilepsy.

\section{MATERIALS AND METHODS}

\section{Object of study}

Studies were conducted on male WAG/Rij rats of 6, 9 and 12 months of age with genetically determined AE $(1,15)$, as well as on Wistar rats of the same age without seizure activity. Animals were kept in standard vivarium conditions under natural light conditions (approximately 8 hours a day), with free access to food and water. In animals of the three age groups, hemodynamics and heart rate variability (HRV) were investigated before and after treatment with the anticonvulsant conjunctival drug, which is used to treat AE. The drug was administered intraperitoneally, 2 times a day at $10 \mathrm{mg} / \mathrm{kg}$, which corresponds to a therapeutic dose. All studies were conducted in strict accordance with the basic bioethical "Rules of work with the use of experimental animals" and ARRIVE (Animal Research: Reporting of In Vivo Experiments) guidelines.

\section{Telemetry monitoring of electrocardiogram (ECG) and electroencephalogram (EEG)}

Recording of the video ECG-EEG was performed in free-moving animals online using the wireless telemetry system ML880B106 by ADInstruments (Australia), which allows to conduct multi-day monitoring without the slightest concern and stress of animals. The signal was transmitted by the transmitter (TR40BB) implanted into the abdominal cavity of the rat. For ECG recording, one of the electrodes of the first pair of the transmitter was fixed to the xiphoid process, the other was fixed to the sternohyoid muscle, which corresponds to the III standard lead. To monitor the total electrical activity of the neocortex (EEG), the registering electrode of the second pair was implanted epidurally over the frontal area of the right hemisphere, where the most pronounced SWD activity is registered, and the reference one was implanted above the cerebellum. Only those spike-wave complexes, whose duration was at least $2 \mathrm{~s}$, were taken into account. Telemetry sensors were implanted two weeks before studying the animals. Operations were performed under general anesthesia with a mixture of Zoletil, Romethar (intraperitoneally at a dose of 20 and $10 \mathrm{mg} / \mathrm{kg}$, respectively), observing the rules of aseptics and antiseptics. After the operation, each animal was kept in a separate cage. During the first 7 days, Gentamicin and Ketonal were administered to the animals.

\section{Telemonitoring of blood pressure in aorta}

The study was performed using a transmitter (TR46SP), which recorded systolic and diastolic blood pressure (BPs, BPd) (Fig. 1). For this purpose, during the operation, the descending part of the aorta was dissected for $1.5-2 \mathrm{~cm}$. Aortic catheterization was performed according to the Seldinger method. Aortic puncture was performed with a cubital catheter with mandrin. Mandrin was taken out and a transmitter catheter was passed through the lumen of the cubital catheter. The catheter was stitched to the muscle and fixed to the aorta with a surgical mesh. The TR46SP transmitter was implanted into the abdominal cavity and it was sutured in layers. For hemostasis, a hemostatic sponge was used. The transmitter's catheter is coated with an anti-platelet substance, so the procedure does not require the use of anticoagulants. Surgical interventions were performed under general anesthesia described above.

Registration and processing of ECG, EEG and blood pressure parameters were performed using the LabChart 7 software for rats. In each research variant, registration was carried out for 5 hours, at night, when the epileptic activity in WAG/Rij rats is 


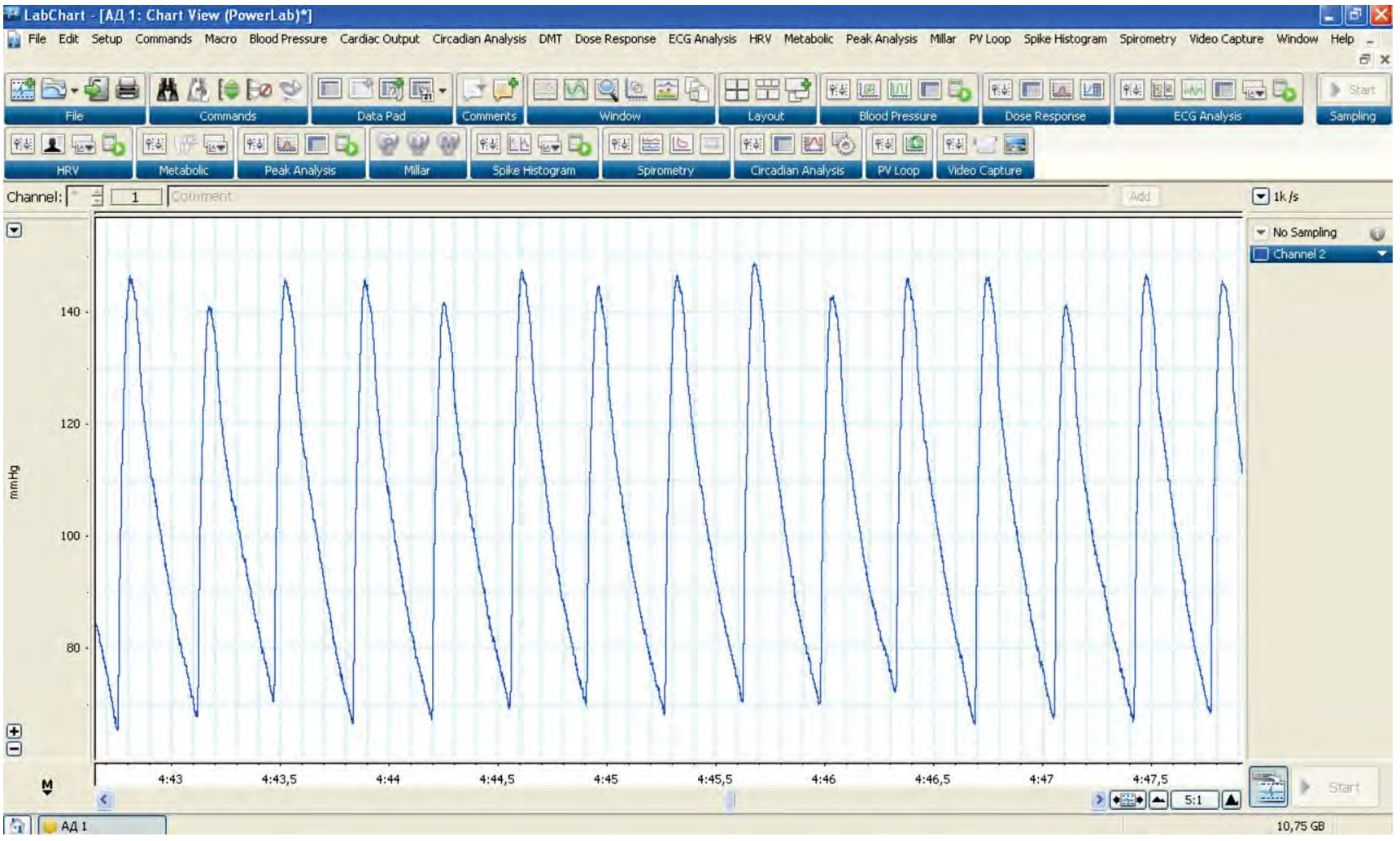

FIGURE 1. Telemetric online recording of blood pressure in the aorta of WAG/Rij rats (LabChart 7 program)

maximal (16). The following was performed to test heart rate variability (HRV): 1) time analysis: heart rate (HR), standard deviation (SDNN), square root of the sum of squares of the difference between the values of consecutive pairs of R-R intervals (RMSSD); 2) spectral analysis: total spectrum power (TP), spectrum power of high frequency (HF), low frequency (LF) and very low frequency (VLF) components with a frequency range of $0.75-3 \mathrm{~Hz}$, 0.02-0.75 Hz, $<0.02 \mathrm{~Hz}$, respectively, spectrum power in the high frequency range in normalized units (HFnu), spectrum power in the low frequency range in normalized units (LFnu), as well as the sympathetic-vagal index (LF/HF). The accuracy of measuring the R-R intervals was $1 \mathrm{~ms}$, the sampling frequency was $1024 \mathrm{~Hz}$. In addition, the duration of left ventricular repolarization (QTc) was analyzed by ECG. EEG analysis allowed us to determine the average number of peak-wave discharges, their average duration, as well as the peak-wave activity index, which reflects the percentage of time taken by SWD during the entire recording period.

\section{Echocardiography (EchoCG)}

Cardiac output (CO) and mean pulmonary arterial pressure (mPAP) were determined by ultra- sound examination of the heart using a Mindray M5 echocardiograph, $10 \mathrm{MHz}$ sensor (Mindray, China). Hemodynamic parameters were calculated according to standard formulas (17). Total peripheral resistance (TPR) - according to the Wetzler-Boger formula $80 \bullet(0.42 \cdot \mathrm{BPs}+0.58 \cdot \mathrm{BPd}) /$ $\mathrm{CO}$; pulmonary vascular resistance $(\mathrm{PVR})-80$ • $\mathrm{mPAP} / \mathrm{CO}$.

\section{Statistical analysis}

Statistical analysis was carried out using the Statistica 10 software. Analysis of the differences between the experimental and control groups was carried out using a single-factor analysis of variance, followed by an evaluation of differences between groups using the Newman-Keyles and Dunn criteria. The verification of the belonging of the samples to the normal distribution was carried out using the Shapiro-Wilk test. In addition, the Pearson correlation coefficient was determined. The results of the study are presented in the form $M \pm$ SEM (mean \pm standard error of mean). Differences with a confidence level of at least $95 \%$ were considered statistically significant. 


\section{RESULTS AND DISCUSSION}

The main indicator of AE in humans and animals is spontaneously arising generalized SWD activity, which serves as an EEG marker of absence epilepsy (Fig. 2). Numerous studies of the neurophysiological mechanisms that provoke the spontaneous emergence of SWD activity in WAG / Rij rats have found similarities to analogous mechanisms in patients with non-convulsive epilepsy $(1,2,15)$. However, spike-wave discharges in rats have frequency $7-10 \mathrm{~Hz}(15)$, which is higher than in humans, $3 \mathrm{~Hz}(2,18)$. In addition, SWD activity in rats appears after puberty and persists throughout life (19), while in humans, spike-wave discharges occur before puberty, and then disappear or transform into other forms of epilepsy (18).

TABLE 1. Age-dependent change in seizure activity in WAG/Rij rats

\begin{tabular}{|l|l|l|l|}
\hline $\begin{array}{l}\text { Age } \\
\text { (months) }\end{array}$ & $\begin{array}{l}\text { Mean } \\
\text { SWD per hour }\end{array}$ & $\begin{array}{l}\text { Total duration (c) } \\
\text { SWD per hour }\end{array}$ & $\begin{array}{l}\text { Peak-wave } \\
\text { index (\%) }\end{array}$ \\
\hline $6(n=10)$ & $19.1 \pm 2.38$ & $51.5 \pm 5.44$ & $1.71 \pm 0.16$ \\
\hline $9(n=10)$ & $29.6 \pm 3.16^{*}$ & $94.7 \pm 10.89^{* *}$ & $2.86 \pm 0.28^{* *}$ \\
\hline $12(n=10)$ & $41.8 \pm 3.91^{*}$ & $135 \pm 12.73^{*}$ & $3.95 \pm 0.35^{*}$ \\
\hline $14(n=10)$ & $39.9 \pm 4.07$ & $127 \pm 11.17$ & $3.67 \pm 0.29$ \\
\hline
\end{tabular}

Note. Comparison of average values in relation to the previous age group. ${ }^{*} \mathrm{P}<0.05,{ }^{* *} \mathrm{P}<0.01$.
As the results of the study showed, an increase in the age of animals with non-convulsive epilepsy is accompanied by an increase in epileptic status. Thus, in 9-month-old WAG/Rij rats, as compared with 6-month-old rats, the average number of SWDs per hour increases by 55\% (Table 1). At 12 months of age, this figure rises by another $41 \%$. And with age, not only the number of SWDs increases, but also their duration. In 9-month-old animals, it is $84 \%$ higher $(\mathrm{P}<0.01)$ than in 6-monthold animals, and in 12-month-old animals it is $43 \%$ higher than in 9-month-old ones. Age-dependent increase in seizure activity is also confirmed by an increase in the peak-wave index. So, in 9-monthold animals peak-wave index is $67 \%$ higher $(\mathrm{P}<0.01)$ than in 6-month-old animals, and it continues to increase until the age of 12 months. A multiple increase in the number of epileptic discharges and their duration in the period from 3 to 12 months of life in WAG/Rij rats was also noted by other authors (15). The results of our research indicate that an increase in status epilepticus occurs up to 12 months of age. In 14-month-old animals, the indices reflecting convulsive activity of the brain were not statistically significantly different from those of 12-month-old rats.

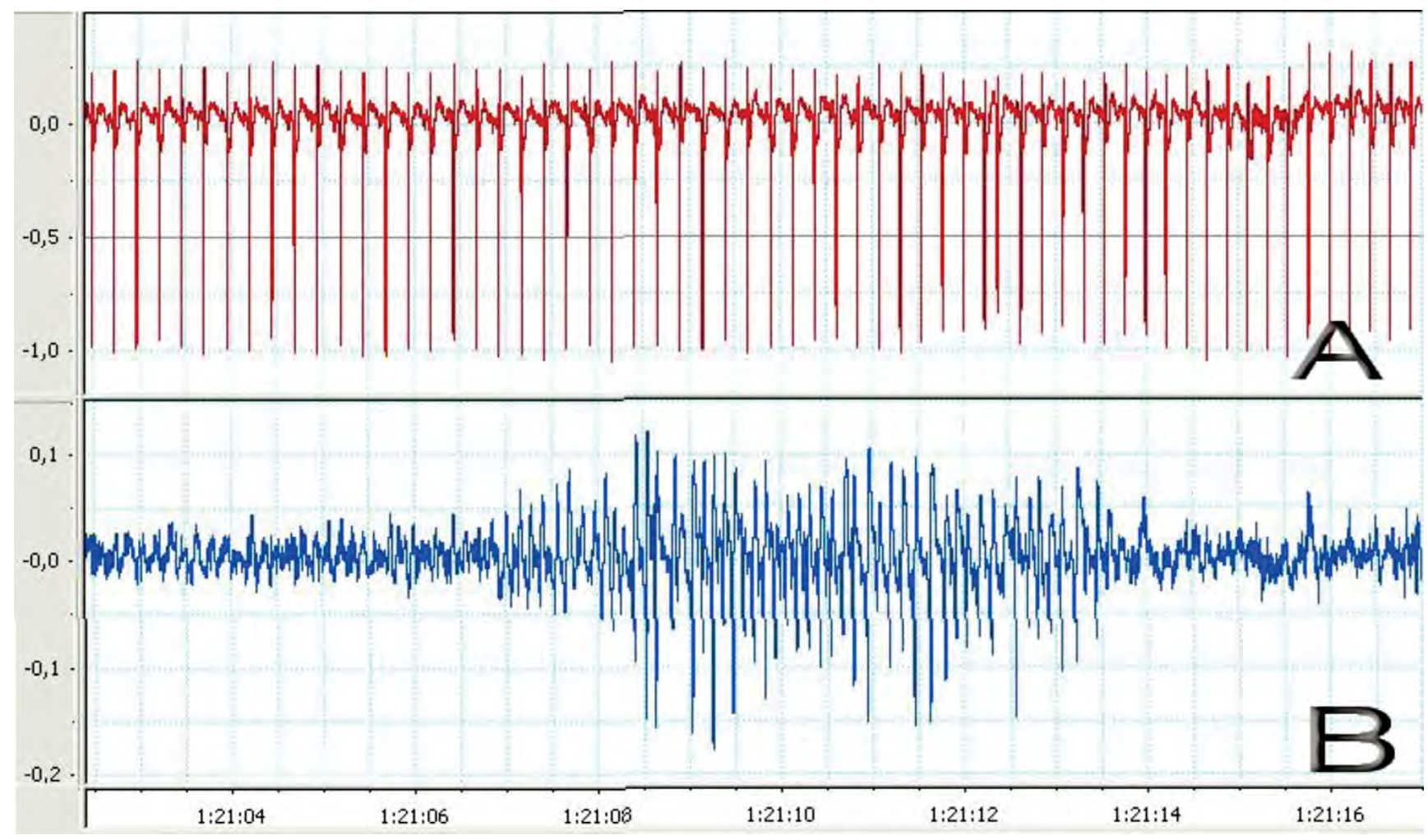

FIGURE 2. Telemetric online registration of ECG $(A)$ and EEG $(B)$ signals. On the EEG there is a typical spontaneous peak-wave discharge in WAG/Rij rats (LabChart 7 program) 
TABLE 2. Changes in heart rate variability and duration of left ventricular repolarization intervals (QTC) in WAG/Rij rats of different age groups before and after Convulex treatment

\begin{tabular}{|c|c|c|c|c|c|c|}
\hline \multirow[b]{2}{*}{ Parameter } & \multicolumn{2}{|c|}{ 6-month-old animals $(n=10)$} & \multicolumn{2}{|c|}{ 9-month-old animals $(n=10)$} & \multicolumn{2}{|c|}{ 12-month-old animals $(n=10)$} \\
\hline & $\begin{array}{l}\text { before } \\
\text { treatment }\end{array}$ & $\begin{array}{c}\text { after } \\
\text { treatment }\end{array}$ & $\begin{array}{l}\text { before } \\
\text { treatment }\end{array}$ & $\begin{array}{c}\text { after } \\
\text { treatment }\end{array}$ & $\begin{array}{c}\text { before } \\
\text { treatment }\end{array}$ & $\begin{array}{c}\text { after } \\
\text { treatment }\end{array}$ \\
\hline $\mathrm{HR}$ & $252 \pm 24,9$ & $274 \pm 23,8$ & $248 \pm 21,8$ & $254 \pm 22,6$ & $276 \pm 27,9$ & $284 \pm 24,1$ \\
\hline SDNN & $10,8 \pm 0,99$ & $15,2 \pm 1,33^{*}$ & $12,8 \pm 1,27$ & $14,1 \pm 1,06$ & $6,8 \pm 0,63$ & $7,2 \pm 0,62$ \\
\hline RMSSD & $4,7 \pm 0,34$ & $6,2 \pm 0,50 *$ & $5,7 \pm 0,52$ & $6,2 \pm 0,49$ & $3,4 \pm 0,27$ & $3,8 \pm 0,28$ \\
\hline $\mathrm{TP}, \mathrm{ms}^{2}$ & $96,1 \pm 7,37$ & $124 \pm 9,14^{*}$ & $67,7 \pm 6,16^{*}$ & $71,7 \pm 5,56$ & $58 \pm 4,80$ & $68,2 \pm 5,28$ \\
\hline $\mathrm{HF}, \mathrm{ms}^{2}$ & $13,5 \pm 1,04$ & $15,8 \pm 1,29$ & $9,5 \pm 0,81^{*}$ & $15,6 \pm 1,43^{* *}$ & $4,9 \pm 0,49^{* *}$ & $6,4 \pm 0,42^{*}$ \\
\hline $\mathrm{LF}, \mathrm{ms}^{2}$ & $41,1 \pm 3,74$ & $35 \pm 2,71$ & $38,5 \pm 3,27$ & $44,8 \pm 4,01$ & $24,6 \pm 1,91^{* *}$ & $28,6 \pm 2,18$ \\
\hline $\mathrm{VLF}, \mathrm{ms}^{2}$ & $42,1 \pm 4,29$ & $72,9 \pm 6,70 \stackrel{* *}{-}$ & $19,7 \pm 1,81^{* *}$ & $11,3 \pm 1,12^{* *}$ & $28,5 \pm 2,72^{*}$ & $33,2 \pm 2,58$ \\
\hline $\mathrm{HF}, \%$ & $13,9 \pm 1,21$ & $12,7 \pm 1,16$ & $14,0 \pm 1,27$ & $21,7 \pm 1,75^{* *}$ & $8,44 \pm 0,64^{* *}$ & $9,38 \pm 0,72$ \\
\hline LF, \% & $42,5 \pm 3,06$ & $28,2 \pm 2,65^{* *}$ & $56,8 \pm 4,99^{*}$ & $62,4 \pm 5,35$ & $42,4 \pm 3,47^{*}$ & $41,9 \pm 3,17$ \\
\hline VLF, \% & $43,5 \pm 3,52$ & $58,9 \pm 4,53$ * & $29,1 \pm 2,67^{*}$ & $15,7 \pm 1,49^{* *}$ & $49,1 \pm 5,0^{* *}$ & $48,6 \pm 4,49$ \\
\hline HF, nu & $24,7 \pm 1,74$ & $31,1 \pm 2,10^{*}$ & $19,7 \pm 1,73$ & $25,8 \pm 1,90^{*}$ & $16,6 \pm 1,58$ & $18,2 \pm 1,57$ \\
\hline $\mathrm{LF}, \mathrm{nu}$ & $75,2 \pm 5,82$ & $68,8 \pm 5,33$ & $80,2 \pm 6,88$ & $74,1 \pm 5,66$ & $83,3 \pm 8,49$ & $81,7 \pm 7,80$ \\
\hline $\mathrm{LF} / \mathrm{HF}$ & $3,04 \pm 0,27$ & $2,21 \pm 0,19^{*}$ & $4,05 \pm 0,33^{*}$ & $2,87 \pm 0,25^{*}$ & $5,02 \pm 0,38$ & $4,46 \pm 0,42$ \\
\hline IC & $6,16 \pm 0,55$ & $6,82 \pm 0,72$ & $6,12 \pm 0,55$ & $3,59 \pm 0,33^{* *}$ & $10,8 \pm 1,13^{* *}$ & $9,65 \pm 0,73$ \\
\hline QTc, ms & $167 \pm 12,9$ & $117 \pm 10,6^{*}$ & $172 \pm 15,8$ & $112 \pm 11,5^{*}$ & $187 \pm 16,3$ & $148 \pm 12,1$ \\
\hline
\end{tabular}

Note. ${ }^{*} \mathrm{P}<0.05,{ }^{* *} \mathrm{P}<0.01$, compared with the previous age group before treatment. ${ }_{-}^{*} \mathrm{P}<0.05, * * \mathrm{P}<0.01$, compared with the respective age group before treatment.

To clarify the issue of the effect of non-convulsive seizure activity on the autonomic regulation of the heart, we conducted monitoring of heart rate variability in animals with different levels of spikewave brain activity. According to the results of the HRV analysis (Table 2), in 9-month-old animals, compared with 6-month-olds, the spectrum power is statistically significantly reduced, and the sympatho-vagal index and the proportion of the low-frequency component in the total spectrum power are increased, which indicates an increase in sympathetic regulation link activity. A further increase in SWD activity in 12-month-old animals is accompanied by a simultaneous decrease $(\mathrm{P}<0.01)$ of both the high-frequency and low-frequency components of HRV, as well as an increase in the ultra-low frequency component. All this reflects deep violations of the mechanisms of not only sympathetic, but also parasympathetic regulation of the functions of the heart. This is confirmed by an increase in the centralization index (IC). Moreover, in 12-monthold animals, compared with 9-month-olds, pulmonary vascular resistance and total peripheral resistance increase by $43 \%$ and $40 \%$, which is respectively $11.2 \pm 1.0710^{3} \mathrm{din} \cdot \mathrm{s}^{\bullet} \mathrm{cm}^{-5}$ and $88.7 \pm$ $8.4210^{3} \mathrm{din} \cdot \mathrm{s}^{\bullet} \mathrm{cm}^{-5}$. In 6-month and 9-month-old animals, hemodynamic parameters did not statistically significantly differ.

It is noteworthy that a high level of CA in all age groups of animals with $\mathrm{AE}$ is accompanied by a sta- tistically significant increase $(\mathrm{P}<0.01)$ in left ventricular repolarization intervals compared to rats of the corresponding age of the Wistar line without seizure activity. This suggests that the violation of the duration of left ventricular repolarization intervals in WAG/Rij rats is associated with epileptic activity. The results obtained are consistent with studies on people with epilepsy, who also have an increase in the QTc interval $(20,21)$.

Thus, ECG monitoring in WAG/Rij animals of 6-12 months of age with absence epilepsy revealed an increase in seizure activity, which is accompanied by an aggravation of changes in HRV. While in Wistar rats of 6-12 months of age, without convulsive pathology, HRV was not statistically significantly different. Comparison of the results of these studies suggests that the level of spike-wave activity affects the nature of the neuro-autonomic regulation of the heart. This is confirmed by the results of studies by a number of authors who found ambiguous changes in HRV in patients with epilepsy $(11,12,22)$. Such differences could be associated with different levels of brain epileptic activity in different patients, especially when they are treated with different antiepileptic drugs.

The introduction of highly effective new-generation drugs into clinical practice has significantly expanded the possibilities of drug therapy for AE. However, some anticonvulsants can disrupt the mechanisms of the autonomic regulation of the 
heart, which also serves as a cause of life-threatening arrhythmias $(14,23,24,25)$.

In this regard, the legitimate question arises: is it possible, by reducing CA with the help of anti-convulsive therapy, to prevent or reduce violations of the autonomic regulation of the heart? To solve it, the Convulex drug used to treat $\mathrm{AE}$ was used. The registration of HRV and echocardiography was carried out after the total duration of SWD activity per hour was reduced $(\mathrm{P}<0.001)$ during treatment with Convulex and remained in each age group at $24.7 \pm$ $2.17 \mathrm{~s}$ (before treatment is indicated in Table 1). In 6-month-old animals, this occurred after $6.52 \pm$ 0.59 days of treatment, in 9-month $-8.71 \pm 0.91$ days, in 12-month $-10.32 \pm 1.01$ days.

Judging by the results of the analysis of HRV, a decrease in the CA of the brain improves the autonomic regulation of the heart in 6- and 9-month-old animals (Table 2). So, after treatment of 6-monthold animals, they increase the total power of the spectrum, the total effect of autonomic regulation (SDNN) and the activity of the parasympathetic link (RMSSD). In this case, there occurs a decrease in the share of the low-frequency component in the total power of the spectrum, as well as the sympatho-vagal index, which reflects a decrease in the sympathetic effect on the heart. Treatment of 9-month-old animals does not increase the reduced power of the spectrum, however, it increases $(\mathrm{P}<0.01)$ the activity of the parasympathetic regulation ( $\mathrm{HF}, \mathrm{ms}^{2}$ and $\mathrm{HF} \%$ ). The resulting decrease in the sympatho-vagal index reflects a decrease in the sympathetic component of the autonomic regulation of the heart rhythm, which improves the functional state of the heart. The obtained data agree with the clinical and experimental studies that show that vagus stimulation increases coronary perfusion, improves autonomic regulation of the heart rate and weakens heart failure in seizure conditions $(22,24)$, while a decrease in vagal activity correlates with an increased risk of sudden cardiac death in epilepsy $(26,27)$. Thus, the results of studies indicate that a decrease in brain CA contributes to the improvement of impaired autonomic regulation of the heart in 6- and 9-month-old animals with $\mathrm{AE}$, and the interval of repolarization of the left ventricle decreases.
Similar improvement in HRV is found in people after their treatment with other anticonvulsants (13). However, the question remains whether the nature of changes in HRV depends on the level of CA of patients. The results of our research have shown that a decrease in brain SWD after treatment with Convulex does not always unambiguously affect HRV in animals of different age groups with absence epilepsy. Thus, anticonvulsant therapy with Convulex reduces peak-wave activity in 12-month-old animals, however, unlike 6-month and 9-month-old rats, does not improve impaired cardiac regulation (Table 2) and does not reduce the increased repolarization interval of the left ventricle.

It is known that disorders of the cardiovascular system, found in animals with non-convulsive epilepsy, are largely associated with impaired central mechanisms of regulation of autonomic functions $(4,16,24)$. Moreover, these disorders are caused by chronic changes in the autonomic centers, which are supported by constantly repeated peak-wave discharges (28). In this regard, the results obtained suggest that treatment of AE with Convulex improves the central mechanisms of the autonomic regulation of the heart. However, if a long-term progressing brain CA exceeds a certain threshold, then the aggravating changes in the autonomic centers of regulation of the cardiovascular system are not restored even after therapeutic reduction of CA.

\section{CONCLUSIONS}

An age-dependent increase in $\mathrm{CA}$ in $\mathrm{WAG} / \mathrm{Rij}$ rats with genetically determined $\mathrm{AE}$ exacerbates changes in hemodynamics and autonomic regulation of heart rhythm. This increases the repolarization interval of the left ventricle, which increases the risk of life-threatening arrhythmias. The decrease in convulsive activity with Convulex makes it possible to improve the autonomic regulation of the heart. However, this possibility persists as long as progressive AE does not exceed a certain threshold. Thereafter, a decrease in brain SWD activity with Convulex does not improve impaired cardiac regulation. 


\section{REFERENCES}

1. Luijtelaar ELJM, Coenen AML. Genetic models of absence epilepsy: New concepts and insights. In Encyclopedia of Basic Epilepsy Res. Oxford: Academic Press. 2009; 1-8.

2. Vol'nova AB, Lenkov DN. Absense epilepsy: mechanisms of hypersynchronization of neural ensembles. Medical academic journal. 2012; 12(1): 7-19.

3. Sitnikova E, Hramov AE, Grubov VV et al. On-off intermittency of thalamocortical oscillations in the electroencephalogram of rats with genetic predisposition to absence epilepsy. Brain Res. 2012; 1436(3): 147-56.

4. Aker RG, Onat FY. Cardiovascular regulation through hypothalamic $\operatorname{GABA}(A)$ receptors in a genetic absence epilepsy model in rat. Epilepsia. 2002; 43(2):107-14.

5. Jansen K, Varon C, Van Huffel S, Lagae L. Cardiac and respiratory autonomic dysfunction in childhood epilepsy. European Journal of Pediatric Neurology. 2013; 17(1): 29-38.

6. Pradhan C, Sinha S, Thennarasu K, Jagadisha T. Quantitative analysis of heart rate variability in patients with absence epilepsy. Neurology India. 2011; 59(1): 25-9.

7. Schuele SU, Widdess-Walsh P, Bermeo A, Lüders HO Sudden unexplained death in epilepsy: the role of the heart. Cleve Clin J Med. 2007; 74(1): 121-27.

8. Terra VC, Cysneiros R, Cavalheiro EA, Scorza FA. Sudden unexpected death in epilepsy: From the lab to the clinic setting. Epilepsy Behavior. 2013; 26(3): 415-20.

9. Cockerall OC, Johnson A, Sander JW. Mortality from epilepsy: results from a prospective population-based study. Lancet. 1994; 344(8927): 918- 21.

10. Tavernor SJ, Brown SW, Tavernor RME, Gifford C. Electrocardiograph QT lengthening associated with epileptiform EEG discharges - a role in sudden unexplained death in epilepsy? Seizure. 1996; 5(1): 79-83.

11. Evrengul $H$, Tanriverdi $H$, Dursunoglu $D$ et al. Time and frequency domain analyses of heart rate variability in patients with epilepsy. Epilepsy Res. 2005; 63(2-3): 131-39.

12. Sayed HL, Kotby, AA, Tomoum HY et al. Non-invasive assessment of cardioregulatory autonomic functions in children with epilepsy. Acta Neurol. Scand. 2007; 115(6): 377-84.

13. Hallioglu O, Okuyaz C, Mert E. Makharoblidze K. Effects of antiepileptic drug therapy on heart rate variability in children with epilepsy. Epilepsy Research. 2008; 79(1): 49-54.

14. Lotufo PA, Valiengo L, Benseñor IM, Brunoni AR. A systematic review and meta-analysis of heart rate variability in epilepsy and antiepileptic drugs. Epilepsia. 2012; 53(2): 272-82.
15. Coenen AML, van Luijtelaar ELJM. Genetic animal models for absence epilepsy: a review of the WAG/Rij strain of rats. Behav. Genet. 2003; 33(6): 635-55.

16. Mamalyga ML, Mamalyga LM. Functional State of the Heart during Spontaneous Nonconvulsive Seizure Activity at Various Times of the Day. Bulletin of Experimental Biology and Medicine. 2016; 161(5): 629-33.

17. Koskenvuo JW, Mirsky R, Zhang Y et al. A comparison of echocardiography to invasive measurement in the evaluation of pulmonary arterial hypertension in a rat model. Int J Cardiovasc Imaging. 2010; 26(5): 509-18.

18. Karlov VA. Absence. Zhurnal nevrologii i psikhiatrii imeni S.S. 2005;105(3): 55-60.

19. Meeren HK, van Luijtelaar ELJM, Lopes da Silva FH, Coenen AM. Evolving concepts on the pathophysiology of absence seizures: the cortical focus theory. Arch Neurol. 2005; 62(3): 371-376.

20. Akalın F, Tırtır A, Yılmaz Y. Increased QT dispersion in epileptic children. Acta Pediatric. 2003; 92(8): 916-20.

21. Brotherstone R, Blackhall $B$, McLellan A. Lengthening of corrected QT during epileptic seizures. Epilepsia. 2010; 51(2): 221-32.

22. Ronkainen E, Korpelainen JT, Heikkinen E et al. Cardiac autonomic control in patients with refractory epilepsy before and during vagus nerve stimulation treatment: a one-year follow-up study. Epilepsia. 2006; 47(3): 556-62.

23. Sevcencu C. Struijk JJ. Autonomic alterations and cardiac changes in epilepsy. Epilepsia. 2010; 51(5): 725-37.

24. Mamalyga ML. Cardiovascular dysfunctions in brain disorders and their treatment. Germany: Academic Publishing, 2017.

25. Lopinto-Khoury C, Mintzer S. Antiepileptic drugs and markers of vascular risk. Curr Treat Options Neurol. 2010; 12(4): 300-8.

26. DeGiorgio Ch. M, Miller P, Meymandi Sh et al. RMSSD, a measure of vagus-mediated heart rate variability, is associated with risk factors for SUDEP: the SUDEP-7 Inventory. Epilepsy Behavior. 2010; 19(1): 78-88.

27. Thayer JF, Yamamoto SS, Brosschot JF. The relationship of autonomic imbalance, heart rate variability and cardiovascular disease risk factors. Int J Cardiol. 2010; 141(2): 122-31.

28. Wasterlain CG, Fujikawa DG, Penix L, Sankar R. Pathophysiological mechanisms of brain damage from status epilepticus. Epilepsia. 1993; 34(1): 37-53. 